\title{
Processed red meat contribution to Irish dietary patterns, defined by cluster analysis
}

\author{
Y.M. Lenighan ${ }^{1}$, A.P. Nugent ${ }^{2}$, K.F. Li ${ }^{2}$, L. Brennan ${ }^{2}$, J. Walton ${ }^{3}$, A. Flynn ${ }^{3}$, H.M. Roche ${ }^{1}$ and \\ B.A. McNulty \\ ${ }^{1}$ Nutrigenomics Research Group, UCD Conway Institute, School of Public Health, Physiotherapy and Sports Science, \\ University College Dublin, Belfield, Dublin 4, Ireland, ${ }^{2} U C D$ Institute of Food and Health, School of Agriculture and \\ Food Science, University College Dublin, Belfield, Dublin 4, Ireland and ${ }^{3}$ School of Food and Nutritional Sciences, \\ University College Cork, Cork, Ireland
}

Processed red meat intakes have been associated with increased risk of cardiovascular disease (CVD) and type 2 diabetes (T2D) ${ }^{(1)}$. Nevertheless, foods are not consumed in isolation and therefore it is important to consider the overall diet when investigating associations between diet and biomarkers of CVD and T2D. The aim of this analysis was to identify dietary patterns, with an emphasis on processed red meat contributions, in a sub cohort $(n=786)$ of the National Adult Nutrition Survey (NANS). The NANS was a crosssectional food consumption survey carried out between 2008 and 2010 in an Irish adult population $(n=1500)$, a four day semiweighted food diary was used to collect habitual food and beverage intakes, and $79 \%$ of participants provided blood samples ${ }^{(2)}$. All meat-containing food codes were assigned to one of four meat food groups $(n=502)$, on the basis of red or white meat, and by processed or unprocessed. Processed meat included products which had undergone salting, curing, fermentation, smoking, flavour enhancement or other preservation processes. The remaining food codes $(n=2050)$ were assigned to 29 food groups, based on those previously identified in an Irish population ${ }^{(3)}$. All food groups were expressed as a percentage contribution to total energy intake (\%TE) and $z$-scores were calculated. Two-step and K-means cluster analysis was applied to derive dietary clusters. Dietary quality was assessed using the Alternate Healthy Eating Index (AHEI) and the Alternate Mediterranean Diet Score (aMED) indices ${ }^{(4)}$.

\begin{tabular}{|c|c|c|c|c|c|c|c|c|c|}
\hline & \multirow{2}{*}{\multicolumn{2}{|c|}{$\frac{\text { Cluster } 1}{n=131}$}} & \multicolumn{2}{|c|}{ Cluster 2} & \multicolumn{2}{|c|}{ Cluster 3} & \multicolumn{2}{|c|}{ Cluster 4} & \multirow[b]{3}{*}{$\mathrm{P}$} \\
\hline & & & \multicolumn{2}{|c|}{$n=70$} & \multicolumn{2}{|c|}{$n=405$} & \multicolumn{2}{|c|}{$n=180$} & \\
\hline & Mean & SD & Mean & SD & Mean & SD & Mean & SD & \\
\hline Processed red meat $(\mathrm{g} / \mathrm{d}){ }^{\S}$ & $25 \cdot 8^{\mathrm{a}}$ & $22 \cdot 9$ & $39 \cdot 2^{\mathrm{b}}$ & $35 \cdot 0$ & $34 \cdot 3^{\mathrm{ab}}$ & $22 \cdot 7$ & $57 \cdot 1^{\mathrm{c}}$ & $46 \cdot 6$ & $<0.001$ \\
\hline Gender $(\mathrm{M} / \mathrm{F} \%)^{\dagger}$ & 34,66 & & 36,64 & & 56,44 & & 57,43 & & $<0.001$ \\
\hline Age (years) $§$ & $48 \cdot 4^{\mathrm{a}}$ & $15 \cdot 7$ & $33 \cdot 4^{\mathrm{b}}$ & $13 \cdot 0$ & $38 \cdot 9^{\mathrm{c}}$ & $15 \cdot 9$ & $48 \cdot 6^{\mathrm{a}}$ & $17 \cdot 2$ & $<0.001$ \\
\hline Energy (kilocalories) $\S$ & $2064^{\mathrm{a}}$ & 569 & $2325^{\mathrm{bc}}$ & 563 & $2422^{\mathrm{b}}$ & 630 & $2237^{\text {ac }}$ & 564 & $<0.001$ \\
\hline AHEI $\Psi^{\top}$ & $34 \cdot 4^{\mathrm{a}}$ & 9.9 & $25 \cdot 7^{\mathrm{b}}$ & $7 \cdot 4$ & $24 \cdot 9^{\mathrm{b}}$ & $8 \cdot 5$ & $21 \cdot 2^{\mathrm{c}}$ & 7.7 & $<0.001$ \\
\hline $\operatorname{aMED}^{\Psi}$ & $3 \cdot 9^{\mathrm{a}}$ & 1.4 & $3 \cdot 0^{\mathrm{b}}$ & 1.6 & $3 \cdot 1^{\mathrm{b}}$ & 1.4 & $2.7^{\mathrm{b}}$ & $1 \cdot 3$ & $<0.001$ \\
\hline$\%$ Total Plasma Fatty Acids & & & & & & & & & \\
\hline Eicosapentaenoic $(\mathrm{C} 20: 5)^{\Psi}$ & $1.83^{\mathrm{a}}$ & 1.42 & $1.27^{\mathrm{ab}}$ & $0 \cdot 61$ & $1 \cdot 26^{\mathrm{b}}$ & $0 \cdot 70$ & $1 \cdot 34^{\mathrm{b}}$ & 0.72 & $<0.001$ \\
\hline Docosahexaenoic $(\mathrm{C} 22: 6)^{\Psi}$ & $3.03^{\mathrm{a}}$ & 1.20 & $2 \cdot 30^{\mathrm{b}}$ & $0 \cdot 81$ & $2 \cdot 30^{\mathrm{b}}$ & $0 \cdot 81$ & $2 \cdot 21^{\mathrm{b}}$ & 0.84 & $<0.001$ \\
\hline
\end{tabular}

$\S$ One-way ANOVA ${ }^{\dagger}$ Pearson chi-squared ${ }^{\Psi}$ General linear model adjusted for age, gender, kilocalories, social class, smoking status, supplement use and fasting status, with a bonferoni correction ${ }^{\text {abcd }}$ Unlike superscripts indicate significant differences between dietary clusters.

Four dietary clusters were derived, cluster 4 presented greater contributions from processed red meat, white bread, butters and potatoes $(\mathrm{P}<0.001)$. Cluster 1 presented the lowest contributions from processed red meat, and higher contributions from fish, fruit and vegetables $(\mathrm{P}<0.001)$. Cluster 2 presented greater contributions from cheese, chips and rice $(\mathrm{P}<0.001)$, while cluster 3 presented higher contributions from unprocessed red meat, alcoholic beverages and savouries $(\mathrm{P}<0.001)$. A range of biomarkers of CVD and T2D were investigated in the current analysis, with no significant differences observed. Plasma levels of C20:5, C22:6, C16:1 and $\mathrm{C} 20: 3$ differed between clusters $(\mathrm{P}<0.001)$. Cluster 1 presented significantly greater plasma levels of $\mathrm{C} 20: 5$ and $\mathrm{C} 22: 6$, which was consistent with greater dietary $\mathrm{C} 20: 5$ and $\mathrm{C} 22: 6$ intakes (\%TE). Dietary patterns with greater processed red meat contributions presented lower LC n-3 PUFA status and poorer dietary quality. Public health strategies are needed to enhance LC n-3 PUFA status, improve dietary quality and reduce disease risk, which could include modification of the fatty acid profile of processed red meat.

This project was funded by FIRM under the NDP 2007-2013.

1. Micha R, Wallace SK \& Mozaffarian D (2010) Circulation $1212271-2283$.

2. National Adult Nutrition Survey Summary Report (2011) Available at: http://t.cn/8s77yIu.

3. Hearty AP \& Gibney MJ (2009) Br J Nutr 101 (4) 598-608.

4. Fung TT, McCullough ML \& Newby PK et al. (2005) Am J Clin Nutr 82 163-173. 